Background 'Time preference' is an economic concept that describes the trade-offs that individuals make between costs and benefits occurring at different points of time. Related psychological concepts include time perspective, impulsivity and delay of gratification. Present orientated people prefer immediate over future gains and place more value on these. Differences in how much value different individuals place on future outcomes are likely to play some role in present day behavioural decisions.

There is substantial cross-sectional evidence that smokers tend to have shorter term time preferences than non-smokers. However, few studies have explored the longitudinal relationship between time preference and smoking cessation.

We explored the relationship between time preference and smoking cessation in a general adult cohort using data from a large Australian longitudinal panel study: the Household Income and Labour Dynamics of Australia (HILDA) survey.

Methods HILDA is a longitudinal, nationally representative, household survey which began in 2001. The focus of the survey is on economic and subjective well-being, and labour market and family dynamics. Data is collected by annual interview and self-complete questionnaire.

Members of the HILDA panel, aged 15-64, who responded to at least four waves of data collection between 2001 and 2008, and reported any level of tobacco consumption at any wave were included in the analyses.

Smoking cessation was measured using self-report questions. Time preference was measured using self-reported time period for financial planning. A range of sociodemographic (age, number of children, various markers of socio-economic position) and smokingrelated (previous quit attempts, years smoked) co-variates were controlled for.

Results 1,817 individuals (representing 7,913 unique observations) were included in the analyses. In univariable analyses, both men and women who reported quitting smoking were more likely to have a longer term time preference in the data collection period immediately prior to quitting than those who did not quit (men: $\mathrm{t}(4,126)=4.59, \mathrm{p}<0.001$; women: $\mathrm{t}(3,783)=7.18, \mathrm{p}<0.001)$. These relationships persisted after control for socio-demographic and smoking-related covariates (hazard ratio of quitting in those with longer vs shorter term time preference ( $95 \%$ confidence intervals) $=1.27$ (1.03 - 1.57) in men; 1.31 (1.05 - 1.63) in women).

Conclusion Adult smokers with a longer term time preference are more likely to quit smoking. This does not exclude the possibility that quitting smoking simultaneously leads to development of longer term time preference. Interventions that encourage development of longer term time preference may enable smoking cessation.

\section{PS36 PROBATION AND NHS WORKING TOGETHER TO REDUCE HEALTH INEQUALITIES: GREAT EXPECTATIONS OR A TALE OF TWO SERVICES}

doi:10.1136/jech-2012-201753.135

J Parkes, P Roderick, S Panozzo. Public Health Sciences \& Medical Statistics, University of Southampton, Southampton, UK

Background Offenders within the community are considered a socially excluded population experiencing poorer health than the general population and difficulties in accessing health care. There is little research focused on Community based offenders supervised by Probation. This study explored probation staff's knowledge, perceptions and attitudes to offender health needs and access to health services.

Methods Using information derived from qualitative research with probation staff and following a pilot, a national on-line questionnaire survey was conducted of probation staff involved in the management of offenders from a geographically representative sample of Probation Trusts in England and Wales. The questionnaire covered: offender health issues, enablers and barriers to health service access, and the NHS/CJS partnership. Models of good/innovative practice were sought.

Results Sixteen Probation Trusts participated and 611/2810 (22\%) responses were obtained. $72 \%$ were female, $58 \%$ had $6-15$ years of experience in probation service. The main offender health issues were substance misuse, mental health $(\mathrm{MH})$, learning disabilities and chronic disease, with alcohol misuse the most important. Provision of easily accessible mental health (for mild -moderate severity) and alcohol treatment services were considered to be inadequate (often/occasional lack of adequate provision 65\% alcohol treatment vs $42 \%$ drug treatment vs $90 \% \mathrm{MH}$ )

The main barrier reported was poor communication between probation and the NHS in particular with Primary Care services. It was felt that many offenders had not registered with a GP and that there was little joint working, understanding and education between primary care and probation. In addition the chaotic lifestyle of offenders lead to them having difficulties in negotiating health services and having appropriate health seeking behaviours, often accessing health services at a point of crisis (A\&E). Responsiveness of health services was perceived to be lacking. Provision of in-house services (particularly mental health) was considered to be very useful where available.

Suggested improvements included undertaking health needs assessments to inform joint strategic planning, focus on improvement of alcohol treatment provision; joint education sessions for GPs and Offender Managers, exploration of in-house mental health services; increased access to health trainers, and greater health focus in probation staff training.

Conclusion Probation staff participants were very supportive of improving health of offenders. This study identified some key barriers to addressing the health needs of offenders and their access to services, with practical solutions identified by participants providing implications for policy within the 'new' NHS, health service commissioning, and future research.

\section{PS37 DOES THE ROUTE TO DIAGNOSIS AFFECT OUTCOMES FOR OESOPHAGO-GASTRIC CANCER PATIENTS}

doi:10.1136/jech-2012-201753.136

${ }^{1} \mathrm{~T}$ Palser, ${ }^{2} \mathrm{D}$ Cromwell, ${ }^{3} \mathrm{R}$ Hardwick, ${ }^{4} \mathrm{~S}$ Riley, ${ }^{5} \mathrm{~K}$ Greenaway, ${ }^{2} \mathrm{~J}$ van der Meulen. ${ }^{1} \mathrm{Clini}-$ cal Effectiveness Unit, Royal College of Surgeons of UK, London, UK; ${ }^{2}$ Department of Health Services Research and Policy, LSHTM, London, UK; ${ }^{3}$ Addenbrookes Hospital, Cambridge, UK; ${ }^{4}$ Northern General Hospital, Sheffield, UK; ${ }^{5}$ The Information Centre for Health and Social Care, Leeds, UK

Background The UK Cancer Reform strategy aims to improve early diagnosis but there is little evidence on how the route to diagnosis and affects outcomes for patients with oesophago-gastric $(\mathrm{O}-\mathrm{G})$ cancer. We investigated the relationship between the route to diagnosis, patient characteristics, treatment intent and one-year survival among $\mathrm{O}-\mathrm{G}$ cancer patients

Methods The study was undertaken in 142 English NHS trusts and 30 cancer networks, with data being prospectively collected on patients diagnosed with O-G cancer between October 2007 and June 2009. Route to diagnosis was defined as general practitioner (GP) referral - urgent or non-urgent, hospital consultant referral, or after an emergency admission. The association between patients' route of diagnosis, treatment intent and survival was estimated using logistic regression.

Results Among 14,102 cancer patients, $66.3 \%$ were diagnosed after a general practitioner (GP) referral, $16.4 \%$ after an emergency admission, and $17.4 \%$ after hospital consultant referral. Of the 9,351 GP referrals, $68.8 \%$ were urgent. Compared to urgent GP referrals, a markedly lower proportion of patients diagnosed after emergency admission had a curative treatment plan (36\% v $16 \%$; 